\title{
A NEW GENERALIZATION OF HARDY-HILBERT'S INEQUALITY WITH NON-HOMOGENEOUS KERNEL
}

\section{Chi-Tung Chang, Jin-Wen LAn And KuO-Zhong WAng}

Abstract. Let $p>1,1 / p+1 / p^{*}=1$, and $a=\left(a_{n}\right)_{n=1}^{\infty}, b=\left(b_{m}\right)_{m=1}^{\infty}$ be two complex sequences. We exhibit the generalization of Hardy-Hilbert's inequality of the following type:

$$
\sum_{n, m \geqslant 1} K\left(\phi_{1}(n), \phi_{2}(m)\right)\left|a_{n}\right|\left|b_{m}\right|<C\left(\sum_{n=1}^{\infty}\left|\frac{a_{n}}{f_{1}\left(\phi_{1}(n)\right)}\right|^{p}\right)^{\frac{1}{p}}\left(\sum_{m=1}^{\infty}\left|\frac{b_{m}}{f_{2}\left(\phi_{2}(m)\right)}\right|^{p^{*}}\right)^{\frac{1}{p^{*}}},
$$

where $K:(0, \infty) \times(0, \infty) \rightarrow(0, \infty), f_{1}, f_{2}, \phi_{1}, \phi_{2}:(0, \infty) \rightarrow(0, \infty)$ and $C$ is a suitable constant. We also get several interesting inequalities which generalize many recent results.

Mathematics subject classification (2010): 26D15, 47B37.

Keywords and phrases: Inequalities, Hilbert's inequality, Hardy-Hilbert's inequality.

\section{REFERENCES}

[1] L. E. AzAR, On some extensions of Hardy-Hilbert's inequality and applications, J. Ineq. Appl., vol. 2008, Article ID 546829, 14 pages, 2008.

[2] I. BRnetić, J. PeČArić, Generalization of inequalities of Hard-Hilbert type, Math. Ineq. Appl., 7 (2004) 217-225.

[3] G. H. Hardy, J. E. Littlewood, G. Pólya, Inequalities, 2nd edition, Cambridge University Press, Cambridge, 1967.

[4] M. KRnić, J. PeČArić, Extension of Hilbert's inequality, J. Math. Anal. Appl., 324 (2006), 150-160.

[5] W. Rudin, Principles of mathematical analysis, 3rd edition, McGraw-Hill, Inc., New York, 1976.

[6] W. Rudin, Real and complex analysis, 3rd edition, Academic Press, New York, 1966.

[7] B. YANG, On a relation between Hilbert's inequality and a Hilbert-type inequality, Appl. Math. Lett., 21 (2008), 483-488.

[8] B. Yang, L. Debnath, On the extended Hardy-Hilbert's inequality, J. Math. Anal. Appl., 272 (2002), 187-199. 